\title{
Central bank intervention and feedback traders
}

\author{
Frank H. Westerhoff* \\ Department of Economics, University of Osnabrück, Rolandstrasse 8, D-49069 Osnabrück, Germany
}

Received 22 April 2002; accepted 28 November 2002

\begin{abstract}
Using a chartist-fundamentalist model we study the effectiveness of linear central bank intervention rules. Leaning against the wind (LAW) dampens the amplitude of exchange rate fluctuations. However, the frequency of cycles may rise due to this policy. Interventions in support of a target exchange rate (TARGET) prove to be unsuccessful. While the amplitude stays constant, the frequency of cycles increases. If this rule is executed with a time lag, the amplitude even grows. Neither of the intervention strategies has an impact on the long-run equilibrium exchange rate.

(C) 2003 Elsevier Science B.V. All rights reserved.

Keywords: Foreign exchange markets; Central bank intervention; Fundamental and technical analysis

JEL classification: E58; F31; G14
\end{abstract}

\section{Introduction}

Central bank interventions are motivated by the desire to check short-run trends or to correct long-term misalignments. Although the empirical literature is ambivalent about the usefulness of such operations, central banks intervene quite frequently in foreign exchange markets (LeBaron, 1999; Neely, 2001; Sarno and Taylor, 2001). At least some policy makers seem to believe that interventions can be an effective tool.

Recently, a new class of exchange rate models has emerged. The chartistfundamentalist approach explores traders who rely on technical and fundamental

* Tel.: +49-541-969-2743; fax: +49-541-969-2742.

E-mail address: fwesterho@oec.uni-osnabrueck.de (F.H. Westerhoff). 
analysis to forecast future prices. Technical trading rules extrapolate past price movements into the future, whereas fundamental trading rules look at the underlying factors. The interaction between these rules may create complex feedback dynamics. For instance, models by Frankel and Froot (1986), Kirman (1991), Brock and Hommes (1998) and Lux and Marchesi (2000) have the potential to replicate the main stylized facts of financial markets quite closely.

Clearly, if exchange rate fluctuations are driven by an endogenous feedback system, central banks may have the opportunity to manipulate the exchange rate path in a way that stabilizes the dynamics. Given the policy importance of central bank intervention, it is surprising that this aspect has received only little attention in the literature so far.

Our aim is to offer a simple framework which may help to understand the working of central bank intervention in the presence of chartists and fundamentalists. Our findings are as follows. Leaning against the wind (LAW) may stabilize the market. On the one hand, it reduces the amplitude of exchange rate fluctuations (i.e. the distortion). On the other hand, the frequency of cycles may rise (i.e. the volatility). Targeting long-run fundamentals has, however, no impact on the amplitude but increases the frequency of cycles. If fundamental targeting is executed with a time lag, the explosiveness of cycles even rises.

\section{The model}

\subsection{Motivation}

Let us briefly sketch the behavioral background of our model. First, traders are assumed to be boundedly rational. Neither do they have access to all relevant information for price determination, nor do they know the mapping from this information to prices. Second, traders follow strict rules. In a broader sense, one may say that our model has an empirical microfoundation. Such a direct underpinning is, for instance, also applied by Lux and Marchesi (2000). As is shown in Hommes, 2001, the behavior of our traders may be consistent with myopic mean-variance maximizers.

Trading volume in spot foreign exchange markets dwarfs that in other financial markets. According to BIS (2002), the overwhelming part of the turnover is due to short-term, speculative trading. Surprisingly, traders strongly rely on simple technical and fundamental trading rules to determine their trading decisions (Taylor and Allen, 1992). Technical analysis is a trading method that attempts to identify trends by inferring future price movements from those of the recent past (for a popular tutorial on technical analysis see Murphy, 1999). Such behavior is not irrational per se. For instance, Szakmary and Mathur (1997) demonstrate that certain technical trading rules may produce excess returns. Fundamental analysis, in turn, presumes that prices have an inherent tendency to converge towards their fundamental value. 


\subsection{Setup}

Our model incorporates four types of agents: market makers, chartists, fundamentalists and a central bank. Market makers quote the exchange rate $S$ for period $t+1$ in response to current excess demand. Assuming a linear price adjustment one obtains

$$
S_{t+1}=S_{t}+\alpha \mathrm{ed}_{t},
$$

where $\alpha$ is a positive reaction coefficient. Excess demand is defined as

$$
\mathrm{ed}_{t}=m d_{t}^{\mathrm{C}}+(1-m) d_{t}^{\mathrm{F}}+d_{t}^{\mathrm{CB}},
$$

where $d^{\mathrm{C}}, d^{\mathrm{F}}$ and $d^{\mathrm{CB}}$ are the demand of chartists, fundamentalists and the central bank, respectively. The market share of chartists (fundamentalists) is denoted by $m$ $(1-m)$.

Chartists use a linear trend forecast to determine their orders

$$
d_{t}^{\mathrm{C}}=\beta\left(S_{t}-S_{t-1}\right),
$$

and fundamentalists expect the exchange rate to move towards its fundamental value $F$

$$
d_{t}^{\mathrm{F}}=\gamma\left(F-S_{t}\right) .
$$

The reaction coefficients $\beta$ and $\gamma$ are both positive.

We focus on the two most common intervention strategies identified empirically (Neely, 2001). The first rule is called "LAW". Using this device, the central bank always trades against past trends and thereby counters the action of chartists. The second rule aims to support a target exchange rate (TARGET). For simplicity, the target rate is assumed to be equal to $F$. By buying an undervalued and selling an overvalued currency, the central bank hopes to push the exchange rate towards its fundamental value. The demand of the central bank may be formalized as

$$
d_{t}^{\mathrm{CB}}=\delta\left(S_{t-1}-S_{t}\right)+\varepsilon\left(F-S_{t}\right),
$$

where $\delta$ and $\varepsilon$ stand for the positive reaction coefficients of the LAW and the TARGET rule, respectively. Note that interventions are routinely sterilized and thus have no direct impact on fundamentals. In addition, interventions are often performed secretly (Neely, 2001). Traders are not able to identify whether a change in exchange rates is triggered by the central bank or by any other factor.

\section{Some analytical results}

For the remainder of this paper, we make two further assumptions: $0 \leq \delta, \varepsilon<$ $m \beta=(1-m) \gamma$. The market impact of central banks' LAW and exchange rate targeting intervention is less than that of chartists and fundamentalists, and the market impact of chartists and fundamentalists is equal. The first restriction seems to be reasonable, since the intervention volume of the central banks is rather low 
compared to the total trading volume in foreign exchange markets (Neely, 2001). The second assumption seems also to be in agreement with empirical observations. For instance, Taylor and Allen (1992) report that for short-term predictions (intraday to 1-week forecasting) the use of technical and fundamental trading rules among professional traders is roughly equal.

Combining Eqs. (1)-(5) yields

$$
S_{t+1}+(\alpha \delta+\alpha \varepsilon-1) S_{t}+(\alpha m \beta-\alpha \delta) S_{t-1}=(\alpha(1-m) \gamma+\alpha \varepsilon) F,
$$

which is a second-order difference equation. As is well known, such a system is capable of generating cyclical motion. Of course, we do not observe deterministic fluctuations in exchange rates. But one should note that simple chartist-fundamentalist models like ours buffeted with random shocks have the potential to produce quite realistic price behavior. Still, the dynamics in such models are mainly driven endogenously through the action of feedback traders (see, for instance, the survey of Hommes (2001)). Our model should thus be interpreted as a stylized world which allows us to determine analytically how central bank interventions affect the exchange rate path in the presence of feedback traders.

Under the assumption that the market impact of chartists is equal to that of fundamentalists $(m \beta=(1-m) \gamma)$, the equilibrium exchange rate in Eq. (6) is equal to the fundamental value perceived by the central bank and fundamental traders. The second-order difference Eq. (6) is stable - that is the exchange rate tends to return to its fundamental value - when $0<\alpha<(m \beta)^{-1}$. Concerning the central bank's intervention policy $(\delta, \varepsilon)$, we are able to show the following (see the appendix for proof):

- The equilibrium exchange rate $\left(S_{t}=F\right)$ is independent of central bank interventions.

- LAW interventions enlarge the area of stability. If the exchange rate path displays converging fluctuations, LAW interventions always diminish the amplitude. However, the frequency of fluctuations increases in regimes in which $\alpha$ exceeds $(2 m \beta)^{-1}$. Unfortunately, this corresponds to periods of high volatility. The LAW rule may well turn out to be a mixed blessing. Although the amplitude declines, more cycles may occur in any given period of time.

- The TARGET rule does not alter the stability region. TARGET interventions have no impact on the amplitude of exchange rate movements but increase the frequency of cycles. If the TARGET rule is executed with a time lag, that is $d_{t}^{\mathrm{CB}}=\varepsilon\left(F-S_{t-1}\right)$, the amplitude even grows. TARGET interventions have no power in stabilizing the dynamics.

\section{Some numerical examples}

Fig. 1 illustrates our analytical findings. The solid lines show the adjustment of the exchange rate after a $1 \%$ shock in the time domain (50 periods). A single shock 


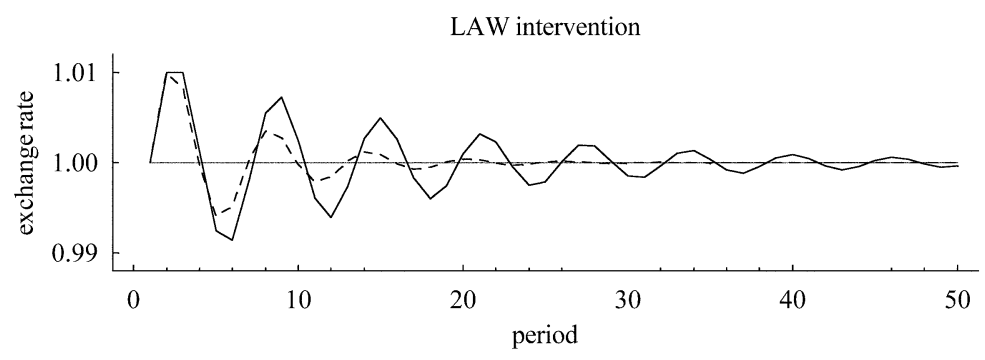

TARGET intervention

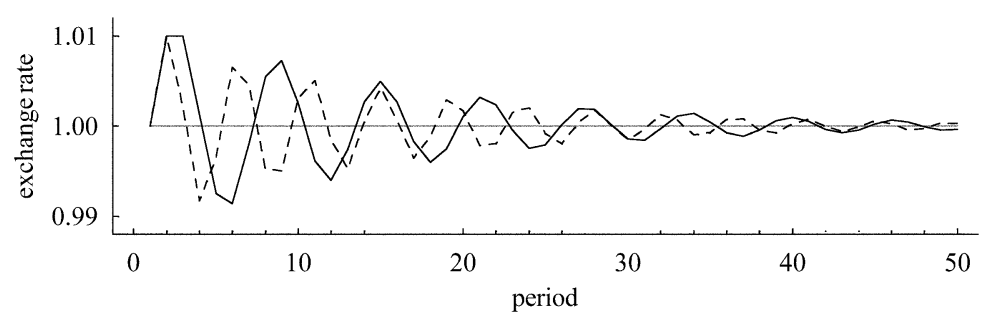

Fig. 1. Examples of intervention operations. The figure shows the exchange rate after a $1 \%$ shock in the time domain (50 observations). Parameters: $\alpha=1.75, \beta=\gamma=1, m=0.5$; solid lines: $\delta=\varepsilon=0$; dashed line in the top: $\delta=0.1$ and $\varepsilon=0$; and dashed line in the bottom: $\delta=0$ and $\varepsilon=0.45$.

triggers lasting fluctuations, which may be interpreted as short-term bubbles. Since the traders generate their own trading signals, volatility is excessive.

The dashed line in the top panel shows the exchange rate path for LAW interventions. This rule clearly decreases the amplitude. However, the frequency of cycles increases slightly (because $\alpha=1.75>(2 m \beta)^{-1}=1$ ). The LAW rule succeeds because it counters the behavior of chartists and weakens their trading signals.

The dashed line in the bottom panel represents the course of the exchange rate for TARGET interventions. The TARGET rule only leads to an increase in the frequency of cycles. The explanation is quite simple. If, for example, the exchange rate converges towards its fundamental value from below, then chartists, fundamentalists and the central bank trade in the same direction. As a result, the exchange rate ascends very quickly and overshoots its fundamental value strongly. Now, this momentum is countered by fundamentalists and the central bank until they all trade in the same direction again.

\section{Conclusions}

On first sight, contracyclical intervention operations appear to be reasonable in the presence of feedback traders. However, our study reveals that simple linear central bank intervention rules may fail in calming down foreign exchange markets. LAW weakens the amplitude of exchange rate swings. Unfortunately, if the market makers react strongly to excess demand — and thereby create a high volatility - the 
LAW rule is likely to intensify the frequency of cycles. Supporting a TARGET only reinforces the frequency of cycles. Moreover, if the target strategy is executed with a time lag, it amplifies the amplitude of the exchange rate path.

\section{Acknowledgements}

I thank Ike Mathur and an anonymous referee for helpful comments and suggestions.

\section{Appendix A}

\section{A.1. Derivation of the fixed point}

Rewrite the time-evolution equation of the exchange rate as

$$
S_{t+1}+X S_{t}+Y S_{t-1}=Z F,
$$

where $X=\alpha \delta+\alpha \varepsilon-1, Y=\alpha m \beta-\alpha \delta$ and $Z=\alpha(1-m) \gamma+\alpha \varepsilon$. Inserting $S=S_{t+1}=$ $S_{t}=S_{t-1}$ into Eq. (7) yields

$$
S+(\alpha \delta+\alpha \varepsilon-1) S+(\alpha m \beta-\alpha \delta) S=(\alpha(1-m) \gamma+\alpha \varepsilon) F .
$$

Due to the assumption $m \beta=(1-m) \gamma$, the fixed point of the model is

$$
S=F \text {. }
$$

According to Eq. (9), the level of the exchange rate is independent of central bank intervention.

\section{A.2. Analysis of the dynamics}

A second-order difference equation is stable if $1+X+Y>0,1-X+Y>0$ and $1-Y>0$. Cycles emerge if $4 Y>X^{2}$. Remember that cycles are already dampened if $1-Y>0$. As demonstrated by Baumol (1961), in the case of dampened cycles a decrease (increase) of $Y$ always yields a lower (higher) amplitude, an isolated decrease (increase) of $X$ reduces (increase) the frequency of cycles and a simultaneous decrease of $Y$ and increase of $X$ increase the frequency of cycles only if $|\Delta X / \Delta Y|$ is larger than $|X / 2 Y|$.

\section{A.2.1. No intervention $(m \beta=(1-m) \gamma>\delta=\varepsilon=0)$}

With no intervention, $\delta$ and $\varepsilon$ are equal to zero. In this case, the three stability conditions discussed above $(1+X+Y>0,1-X+Y>0$ and $1-Y>0)$ are given as

$$
\begin{aligned}
& 1-1+\alpha m \beta>0 \text { or } \alpha>0, \\
& 1+1+\alpha m \beta>0 \text { or } \alpha>-(0.5 m \beta)^{-1}, \\
& 1-\alpha m \beta>0 \text { or } \alpha<(m \beta)^{-1} .
\end{aligned}
$$


Together, Eqs. (10)-(12) restrict the area of stability to

$$
0<\alpha<(m \beta)^{-1} .
$$

Cycles emerge for

$$
4 \alpha m \beta>(-1)^{2} \text { or } \alpha>(4 m \beta)^{-1} \text {. }
$$

Combining Eqs. (12) and (14) shows that converging fluctuations occur for

$$
(4 m \beta)^{-1}<\alpha<(m \beta)^{-1} \text {. }
$$

\section{A.2.2. LAW intervention $(m \beta=(1-m) \gamma>\delta>0=\varepsilon)$}

With LAW intervention but no fundamental targeting intervention, $\delta$ is positive and $\varepsilon$ is equal to zero. From the stability conditions one obtains

$$
\begin{aligned}
& 1+a \delta-1+\alpha m \beta-a \delta>0 \text { or } \alpha>0, \\
& 1-a \delta+1+\alpha m \beta-a \delta>0 \text { or } \alpha>(\delta-0.5 m \beta)^{-1} \text { for } \delta<0.5 m \beta \text { and } \alpha \\
& \quad<(\delta-0.5 m \beta)^{-1} \text { for } \delta>0.5 m \beta, \\
& 1-\alpha m \beta+a \delta>0 \text { or } \alpha<(m \beta-\delta)^{-1} .
\end{aligned}
$$

The first part of Eq. (17) requires $\alpha$ to be positive. The second part of Eq. (17) intersects Eq. (18) from above at $\delta=0.75 \mathrm{~m} \beta$. The solution is thus stable for

$$
\begin{aligned}
0 & <\alpha<(m \beta-\delta)^{-1} \text { for } 0<\delta<0.75 m \beta \text { and } 0<\alpha<(\delta-0.5 m \beta)^{-1} \text { for } 0.75 m \beta \\
& <\delta<m \beta .
\end{aligned}
$$

Since the upper bound of Eq. (19) is larger than the upper bound of Eq. (13) for $0<d<m \beta$, LAW interventions enlarge the area of stability.

The model generates fluctuations for

$$
4(\alpha m \beta-\alpha \delta)>(\alpha \delta-1)^{2} \text { or } \frac{-1}{\delta^{2}}>\alpha^{2}+\alpha\left(\frac{2}{\delta}-\frac{4 m \beta}{\delta^{2}}\right) .
$$

Solving Eq. (20) for $\alpha$ yields the condition

$$
\frac{2 m \beta-\delta-2 \sqrt{m^{2} \beta^{2}-m \beta \delta}}{\delta^{2}}<\alpha<\frac{2 m \beta-\delta+2 \sqrt{m^{2} \beta^{2}-m \beta \delta}}{\delta^{2}} .
$$

The left-hand side of Eq. (21) is located between 0 and Eq. (18). Furthermore, the right-hand side of Eq. (21) intersects Eq. (18) at $\delta=0.75 \mathrm{~m} \beta$ from above (to see this, substitute $\delta=x m \beta$ with $0<x<1$ in Eqs. (18) and (21)). Summing up, dampened cycles are observed in the region 


$$
\begin{aligned}
& \frac{2 m \beta-\delta-2 \sqrt{m^{2} \beta^{2}-m \beta \delta}}{\delta^{2}}<\alpha<\frac{1}{m \beta-\delta} \text { for } 0<\delta \\
& <0.75 m \beta \text { and } \frac{2 m \beta-\delta-2 \sqrt{m^{2} \beta^{2}-m \beta \delta}}{\delta^{2}}<\alpha \\
& <\frac{2 m \beta-\delta+2 \sqrt{m^{2} \beta^{2}-m \beta \delta}}{\delta^{2}} \text { for } 0.75 m \beta<\delta<m \beta .
\end{aligned}
$$

Note that $Y$ declines in $\delta$. Thus, if Eq. (22) holds, LAW interventions diminish the amplitude of the cycles. Since a shift in $\delta$ causes $|\Delta X / \Delta Y|=|\alpha /(-\alpha)|=1$ and since $|X / 2 Y|=|-1 /(2 \alpha m \beta)|$ at $\delta=0$, the frequency of fluctuations increases for $\alpha>$ $(2 m \beta)^{-1}$.

\section{A.2.3. TARGET intervention $(m \beta=(1-m) \gamma>\varepsilon>0=\delta)$}

With fundamental targeting intervention but no LAW intervention, $\varepsilon$ is positive and $\delta$ is equal to zero. In this case, the exchange rate path is stable for

$$
\begin{aligned}
& 1+\alpha \varepsilon-1+\alpha m \beta>0 \text { or } \alpha>0, \\
& 1-\alpha \varepsilon+1+\alpha m \beta>0 \text { or } \alpha>-(0.5 m \beta-0.5 \varepsilon)^{-1}, \\
& 1-\alpha m \beta>0 \text { or } \alpha<(m \beta)^{-1} .
\end{aligned}
$$

From Eqs. (23) and (25) it follows that the stability region is bounded to

$$
0<\alpha<(m \beta)^{-1} \text {. }
$$

Since Eq. (26) is equivalent to Eq. (13), TARGET interventions do not alter the stability area.

Cycles result for

$$
4 \alpha m \beta>(\alpha \varepsilon-1)^{2} \text { or } \frac{-1}{\varepsilon^{2}}>\alpha^{2}-\alpha\left(\frac{2}{\varepsilon}+\frac{4 m \beta}{\varepsilon^{2}}\right) .
$$

Rearranging Eq. (27) in terms of $\alpha$ delivers

$$
\frac{2 m \beta+\varepsilon-2 \sqrt{m^{2} \beta^{2}+m \beta \varepsilon}}{\varepsilon^{2}}<\alpha<\frac{2 m \beta+\varepsilon+2 \sqrt{m^{2} \beta^{2}+m \beta \varepsilon}}{\varepsilon^{2}} .
$$

The left-hand side of Eq. (28) is positive. Condition (25) lies between the left-hand side and the right-hand side of Eq. (28) (use again $\delta=x m \beta$ with $0<x<1$ in Eqs. (25) and (28) for verification). Hence, the stability of the fluctuations is ensured for

$$
\frac{2 m \beta+\varepsilon-2 \sqrt{m^{2} \beta^{2}+m \beta \varepsilon}}{\varepsilon^{2}}<\alpha<\frac{1}{m \beta} .
$$

Since $\varepsilon$ is not part of $Y$, the amplitude stays constant. But $X$ increases in $\varepsilon$. Thus, 
TARGET interventions intensify the frequency of cycles. For $d_{t}^{\mathrm{CB}}=\varepsilon\left(F-S_{t-1}\right)$, it is straightforward to check that the amplitude grows $(X=-1$ and $Y=\alpha m \beta+\alpha \varepsilon)$.

\section{References}

Baumol, W., 1961. Pitfalls in contracyclical policies: some tools and results. Rev. Econ. Stat. 435, 21-26. BIS, 2002. Triennial central bank survey: foreign exchange and derivatives market activity in 2001, BIS, Basel.

Brock, W., Hommes, C., 1998. Heterogeneous beliefs and routes to chaos in a simple asset-pricing model. J. Econ. Dyn. Control 22, 1235-1274.

Frankel, J., Froot, K., 1986. Understanding the US dollar in the eighties: the expectations of chartists and fundamentalists. Econ. Rec. 62, 24-38.

Hommes, C., 2001. Financial markets as nonlinear adaptive evolutionary systems. Quant. Finance 1, 149167.

Kirman, A., 1991. Epidemics of opinion and speculative bubbles in financial markets. In: Taylor, M. (Ed.), Money and Financial Markets. Blackwell, Oxford, pp. 354-368.

LeBaron, B., 1999. Technical trading rule profitability and foreign exchange intervention. J. Int. Econ. 49, $125-143$.

Lux, T., Marchesi, M., 2000. Volatility clustering in financial markets: a micro-simulation of interacting agents. Int. J. Theor. Appl. Finance 3, 675-702.

Murphy, J., 1999. Technical Analysis of Financial Markets. New York Institute of Finance, New York.

Neely, C., 2001. The practice of central bank intervention: looking under the hood. Federal Reserve Bank of St. Louis Rev. 83, 1-10.

Sarno, L., Taylor, M., 2001. Official intervention in the foreign exchange market: is it effective and, if so, how does it work? J. Econ. Lit. 39, 839-868.

Szakmary, A., Mathur, I., 1997. Central bank intervention and trading rule profits in foreign exchange markets. J. Int. Money Finance 16, 513-535.

Taylor, M., Allen, H., 1992. The use of technical analysis in the foreign exchange market. J. Int. Money Finance 11, 304-314. 